\title{
Intereses y perspectiva sobre la carrera de medicina: un contraste entre estudiantes de medicina de primero y séptimo año
}

\author{
ALBERTO TOSO ${ }^{1, a}$, MARÍA JOSÉ AYALA ${ }^{2, a}$, VALENTINA BRUNNER ${ }^{3, b}$, \\ JOSEFINA RODRÍGUEZ ${ }^{1, \mathrm{~b}}$, MARÍA ISABEL HERNÁNDEZ4, \\ CINTHYAURQUIDI ${ }^{5}$, VERÓNICA MERICQ ${ }^{4}$
}

\section{Interests and perspectives of first and last year medical students}

Background: The interests that motivate medical students to study this career are diverse and they may change during the seven years of study. In Chile, 22 universities offer medicine and the number of graduated students has increased by more than $50 \%$ over the last 10 years. Aim: To determine the motivational profile of medical students at admission, and at the end of their career. Subjects and Methods: A voluntary anonymous survey was applied to 275 first and 140 seventh year medical students from one traditional public and two private schools. Results: The main reason for applying to medical school was social interest (68.7\%), followed by interest in science and academia. Thirty six percent of students from seventh year would not study medicine again. In the seventh year, the interest in medical care persists in $88 \%$ of students, followed by academic interests in 64\%. Only 24\% had research interests. Fifty nine and 57\% of students projected their medical work in private and public hospital settings, respectively. Only $11 \%$ projected themselves as doing research. Sixty nine percent of students would like to receive more information about post graduate education. Conclusions: There is a low interest in research and a high percentage of seventh year students that would not apply to medicine again. Medical schools should perform a systematic analysis of students' interests to improve faulty areas.

(Rev Med Chile 2012; 140: 609-615).

Key words: Career choice; Education, medical; Students, medical.

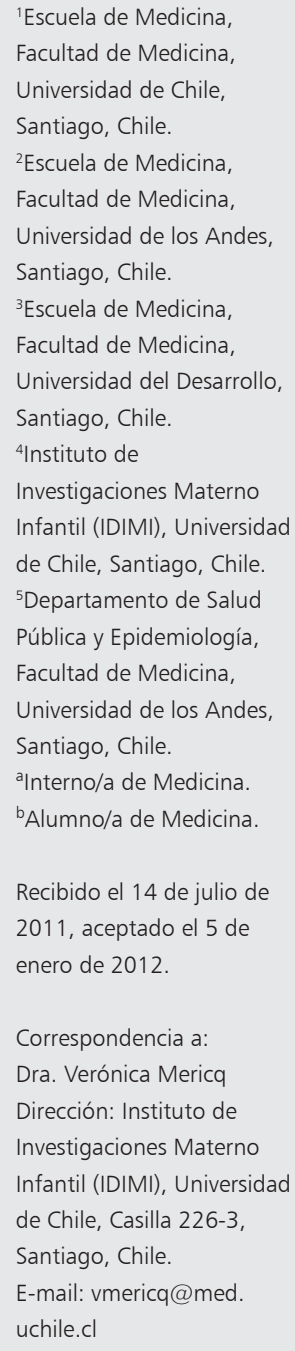

L os intereses y perspectivas de los alumnos de medicina de nuestro país son importantes ya que son las próximas generaciones de médicos las que van a determinar las políticas relacionadas a la formación de profesionales del sector de salud nacional.

Estudios en Estados Unidos de Norteamérica demuestran que los intereses de los médicos han cambiado a través de los años: disminuyendo el por actividades académicas y aumentando el por actividades asistenciales. En un estudio entre los años 1985 y 2003 en recién egresados, se observó que dentro de la actividad académica, docencia se mantuvo estable, y la investigación mostró una baja $(4,6 \% \text { versus } 1,8 \%)^{1}$.

Por otra parte, dentro del interés asistencial, este se ha enfocado hacia la práctica privada, con interés en especialidades y subespecialidades más "rentables". Las razones esgrimidas para este cambio, de acuerdo a un estudio realizado a residentes de dermatología en Estados Unidos de Norteamérica son: burocracia en el desarrollo de la carrera académica $(63,2 \%)$, menor renta $(52,6 \%)$, la ausencia de un mentor o un guía en la carrera (50\%) y el ambiente de trabajo $(26,3 \%)^{2}$.

En Chile, desde el año 1991 el número de 
escuelas de medicina ha aumentado de 6 a 28, en 22 universidades (incremento de $467 \%)^{3}$. Lo que ha incrementado el número de médicos recién egresados desde 760 en el año 2003 a 1.184 en el año 2010, representando un alza de $55 \%{ }^{4}$.

Dado el advenimiento de nuevas escuelas de medicina en nuestro país y la presencia creciente de clínicas privadas, podría esperarse que el perfil motivacional de los alumnos cambiara. Esto nos motivó a estudiar el perfil inicial de estudiantes de medicina de universidades chilenas y conocer su interés, motivación y perspectivas al ingreso y al final de su carrera.

\section{Sujetos y Métodos}

Este estudio es transversal y descriptivo con el objeto de conocer los intereses, motivaciones y perspectivas de los estudiantes de medicina. Se utilizó una encuesta anónima, autoaplicada, fácil de contestar, similar a otras encuestas utilizadas ${ }^{5,6}$, pero abreviada y ajustada a nuestra realidad. La encuesta estaba constituida por 7 preguntas, de selección múltiple, donde se podía marcar más de una respuesta (Figura 1). Se solicitó colaboración voluntaria y se aseguró manejo confidencial de la información.

La encuesta se aplicó a 415 estudiantes de medicina de primero (275) y séptimo año (140), pertenecientes a dos universidades privadas (U1 y U2) (239 alumnos) y una universidad tradicional pública (U3) (176 alumnos), durante los períodos 2009 y 2010. De los estudiantes de primero 76 corresponden a U1, 78 a U2 y 121 a U3, mientras que de los estudiantes de séptimo 30 corresponden a U1, 55 a U2 y 55 a U3. Las tres universidades pertenecen a la ciudad de Santiago de Chile.

Toda la información fue procesada y analizada mediante el software estadístico STATA IC/ v11.0 ${ }^{\circledR}$. La descripción de los datos se hizo a través de frecuencias y proporciones. Se comparó las múltiples proporciones de respuestas dentro de cada pregunta, entre $1^{\circ}$ y $7^{\circ}$ año y entre universidades. La comparación de proporciones entre dos grupos, se hizo a través de la prueba $\chi^{2}$ para muestras independientes, se ajustó modelos de regresión logística para la comparación de más de tres grupos. Se consideró como estadísticamente significativo un $\mathrm{p}$ value $<0,05$.

\section{Resultados}

Las encuestas fueron respondidas por todos los alumnos a quienes se les entregó, que representan aproximadamente $84 \%$ de los alumnos de $1^{\circ}$ año y $42,3 \%$ de los alumnos cursando $7^{\circ}$ año en las escuelas incluidas, durante un año académico.

\section{Motivación de los estudiantes por ingresar a estudiar medicina}

El principal motivo de ingreso en ambos cursos fue el interés social, seguido por el interés por la ciencia y por lo académico. En ambos cursos la primera preferencia fue el interés social, pero sólo en los alumnos de primer año fue mayor a la segunda preferencia $(p<0,01)$. La segunda preferencia varió según el curso, observándose en séptimo año un incremento en el interés por lo académico. Por otra parte, el interés por la ciencia fue mayor en los alumnos de primer año (Tabla 1). No se observaban diferencias entre las distintas universidades.

El 36,4\% ( $\mathrm{n}=51)$ de los alumnos de séptimo año encuestados $(\mathrm{n}=140)$, correspondientes al $43,3 \%$ de los alumnos de U1 ( $\mathrm{n}=13), 40 \%$ de los alumnos de U2 $(\mathrm{n}=22)$ y al $29,1 \%$ de los alumnos de U3 $(n=16)$, respondió que no volvería a estudiar medicina. No existió diferencia entre las distintas universidades, a pesar de que se observó un porcentaje menor de alumnos en U3 que respondieron negativamente a esta pregunta (Figura 2).

En la encuesta se daba espacio a los alumnos de $7^{\circ}$ año para indicar las razones esgrimidas para seguir o no motivado. De los alumnos que respondieron negativamente a esta pregunta $(\mathrm{n}=51)$, el argumento más frecuente correspondió al sacrificio de tiempo con insuficiente desarrollo de otras áreas $(64,7 \%)$. Con menor frecuencia se mencionaron, lo extenso de la carrera, la preferencia por otras carreras diferentes al área de salud, desilusión, y las escasas perspectivas de alcanzar una opción de especialización en áreas de interés.

Respecto al interés de todos alumnos encuestados observamos que predominaba lo asistencial $(87,7 \%)$, seguido por lo académico $(63,6 \%)$ y finalmente la investigación, $(23,6 \%)(\mathrm{p}<0,01)$. Al comparar ambos cursos, existe un aumento por el interés académico en alumnos de $7^{\circ}$ año $(58,4 \%$ vs $73,8 \%)$. Al analizar estos datos por universidad, la U1 fue la única que mostró un aumento en interés 


\section{ENCUESTA ANÓNIMA A ALUMNOS DE ESCUELAS DE MEDICINA DE CHILE}

UNIVERSIDAD

CURSO: $1^{\circ} \mathrm{MEDICINA}$

CIUDAD DE ORIGEN:

$7^{\circ} \mathrm{MEDICINA}$

FAMILIARES MÉDICOS: NO SI QUIEN:

Puedes marcar más de una alternativa en cada pregunta. Recuerda que esta encuesta es anónima.

1. ¿QUÉ MOTIVO TU INGRESO A ESTUDIAR MEDICINA?
a) Interés por la ciencia.
b) Buen puntaje en PSU
c) Presión familiar
d) Interés académico
e) Interés social (ayuda)
f) Buena reputación de la carrera.
g) Status en la sociedad
h) Proyección económica

PARA LOS ALUMNOS DE 7: SI ESTUVIERAS NUEVAMENTE FRENTE A LA DECISIÓN DE QUÉ ESTUDIAR: ELEGIRÍAS MEDICINA SI_ NO_ POR QUÉ?

2. ¿CUÁL ES TU INTERÉS ACTUAL?
a) Asistencial
b) Académico
c) Investigación
d) $A+b$
e) $A+C$
f) $B+C$
g) todos

3. ¿CÓMO PROYECTAS TU CARRERA DE MÉDICO?
a) Trabajando en una clínica
b) Salud pública
c) Trabajando en un consultorio
d) Trabajando en una universidad
e) Sólo práctica privada
f) Haciendo investigación

4. ¿A QUÉ ESPECIALIDAD TE GUSTARÍA DEDICARTE?
a) General de zona
b) Medicina general o familiar
c) Medicina Interna
d) Pediatría
e) Cirugía (General__ Oftalmología__ ORL__ Traumatología_ )
f) Ginecología y obstetricia
g) Salud Pública-administración en salud.

5. ¿SI PUDIERAS OPTAR A UNA SUB-ESPECIALIDAD QUE TE GUSTARÍA?

6. ¿POR QUÉ TE GUSTARÍA HACER UNA SUB-ESPECIALIDAD?
a) Perfeccionarme profesionalmente
b) Mayores oportunidades laborales
c) Mejores oportunidades económicas
d) Status profesional
e) Otra:

7. ¿DE QUÉ TE GUSTARÍA RECIBIR MÁS INFORMACIÓN DURANTE LA CARRERA?
a) Proyección laboral
b) Proyección económica
c) Orientación a especialidad-subespecialidad
d) Posibilidades de perfeccionamiento
e) Otra

Muchas Gracias!!

Figura 1. Encuesta anónima realizada a los estudiantes de medicina de primer y séptimo año. 
académico. En alumnos de séptimo año de la U3 el interés asistencial siguió siendo mayor $(\mathrm{p}<0,01)$. Por otra parte, se pudo constatar una tendencia a la disminución del interés por la investigación entre los alumnos de $7^{\circ}$ respecto a los de $1^{\circ}$. El interés asistencial se mantuvo durante todos los años de carrera en todas las escuelas encuestadas (Tabla 2).

\section{Proyección profesional de los alumnos} de $1^{\circ} y 7^{\circ}$ año

La mayoría de los estudiantes se proyecta trabajando en un ambiente hospitalario (Figura 3), sin diferencias entre los ámbitos privado $(59,5 \%)$ o el público terciario $(57,1 \%)$. A su vez, estas dos preferencias se mostraron mayores que el resto de las opciones, tanto por universidad como por curso $(\mathrm{p}<0,01)$. En el primer año de medicina

Tabla 1. Motivación de los estudiantes para ingresar a estudiar medicina, estudiantes de primer año vs estudiantes de séptimo año

\begin{tabular}{|lcc|}
\hline Motivación & $\begin{array}{c}\mathbf{1}^{\circ} \text { Medicina } \\
(\mathbf{n}=\mathbf{2 7 5})\end{array}$ & $\begin{array}{c}\mathbf{7}^{\circ} \text { Medicina } \\
(\mathbf{n}=\mathbf{1 4 0})\end{array}$ \\
\hline Interés por la ciencia & $50,3 \%$ & $38,3 \% *$ \\
\hline Buen puntaje PSU & $9,8 \%$ & $14,8 \%$ \\
\hline Presión Familiar & $1,8 \%$ & $6,3 \% *$ \\
\hline Interés académico & $41,2 \%$ & $51,7 \% *$ \\
\hline Interés social (ayuda) & $72,3 \%$ & $62,4 \% *$ \\
\hline Status en la sociedad & $16,8 \%$ & $18,4 \%$ \\
\hline Proyección económica & $9,1 \%$ & $7,8 \%$ \\
\hline
\end{tabular}

${ }^{*} p<0,05$. los alumnos no muestran diferencias respecto a la preferencia de proyección laboral en un consultorio o en una universidad.

Al comparar a las preferencias entre alumnos de $1^{\circ}$ y $7^{\circ}$ año, se observó una disminución del porcentaje que se proyecta trabajando en salud pública terciaria $(\mathrm{p}<0,05)$ acompañado de un aumento de interesados en trabajar en un consultorio y/o en universidad $(\mathrm{p}<0,05)$.

Si se analiza por universidad, existió un aumento de proyección en salud pública terciaria en $7^{\circ}$ año respecto a $1^{\circ}(87,1$ versus $60,5 \%, p<0,05)$ en U1 y en U3 por consultorio.

Tabla 2. Interés actual de los alumnos de medicina, estudiantes de primer año vs estudiantes de séptimo año

\begin{tabular}{|ccccc|}
\hline & & $\begin{array}{c}\text { Interés } \\
\text { asistencial }\end{array}$ & $\begin{array}{c}\text { Interés } \\
\text { académico }\end{array}$ & $\begin{array}{c}\text { Interés en } \\
\text { investigación }\end{array}$ \\
\hline $1^{\circ}$ U1 & $88,2 \%$ & $61,8 \% *$ & $31,6 \%$ \\
& U2 & $92,2 \%$ & $64,9 \%$ & $22,1 \%$ \\
U3 & $82,6 \%$ & $52,1 \%$ & $24,8 \%$ \\
& Total & $86,9 \%$ & $58,4 \%{ }^{*}$ & $25,9 \%$ \\
$7^{\circ}$ U1 & $96,8 \%$ & $87,1 \% *$ & $19,4 \%$ \\
& U2 & $81,8 \%$ & $78,2 \%$ & $20 \%$ \\
U3 & $92,7 \%$ & $61,8 \%$ & $18,2 \%$ \\
Total & $89,4 \%$ & $73,8 \%{ }^{*}$ & $19,1 \%$ \\
\hline
\end{tabular}

Alumnos primer año: U1:76, U2:78, U3:121, Total: 275. Alumnos séptimo año: U1:30, U2:55, U3:55, Total: 140. *Alumnos de $1^{\circ}$ y $7^{\circ}$ en $U 1$ respecto a interés académico $(p<0,05)$. \#Alumnos de $1^{\circ}$ y $7^{\circ}$ año respecto a interés académico $(p<0,05)$.

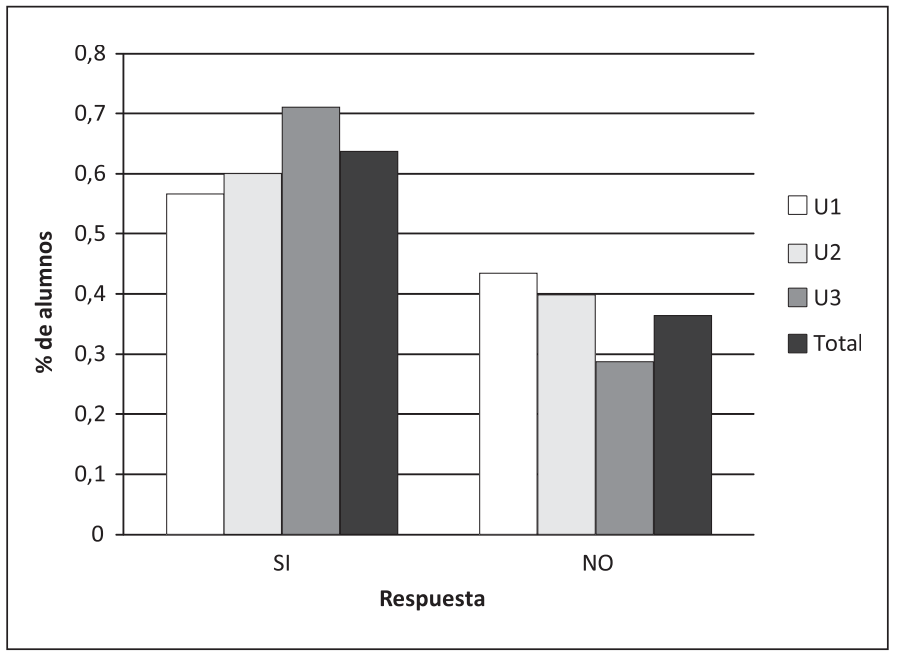

Figura 2. Respuesta a la pregunta ¿Volverías a estudiar medicina? en los alumnos de $7^{\circ}$ año. Total de alumnos de $7^{\circ}$ año $\mathrm{n}=140 ; \mathrm{U} 1=30$, $U 2=55, \cup 3=55$. Un $63,6 \%(n=89)$ de los alumnos encuestados, distribuidos en 17 alumnos de U1, 33 alumnos de U2 y 39 alumnos de U3, respondió afirmativamente sin diferencias entre cada universidad. Un $36,4 \%(n=51)$ de los alumnos encuestados, distribuidos en 13 alumnos de U1, 22 alumnos de U2 y 16 alumnos de U3, respondió negativamente sin diferencias entre cada universidad. 
En la preferencia de especialización, los alumnos de $7^{\circ}$ año de U3 prefiere mayoritariamente postular a médico general de zona $(20 \%$ vs otras universidades $<4 \%, \mathrm{p}<0,05)$.

\section{Información adicional deseable durante la carrera de medicina}

Destaca, el deseo de tener una mayor orientación respecto a especializaciones $(69,2 \%)$ $(\mathrm{p}<0,01)$ sin diferencias por curso. Luego, las preferencias se inclinan por posibilidad de perfeccionamiento $(44,1 \%)$ y proyección laboral $(39,5 \%)$, observándose diferencias entre estas dos preferencias en los alumnos de séptimo año (Figura 4). Los alumnos de $7^{\circ}$ desean mayor información sobre proyección laboral respecto a los de $1^{\circ}(\mathrm{p}<0,05)$. Al analizar esta parte de la encuesta por universidad, sólo en la U1 se observó un au-

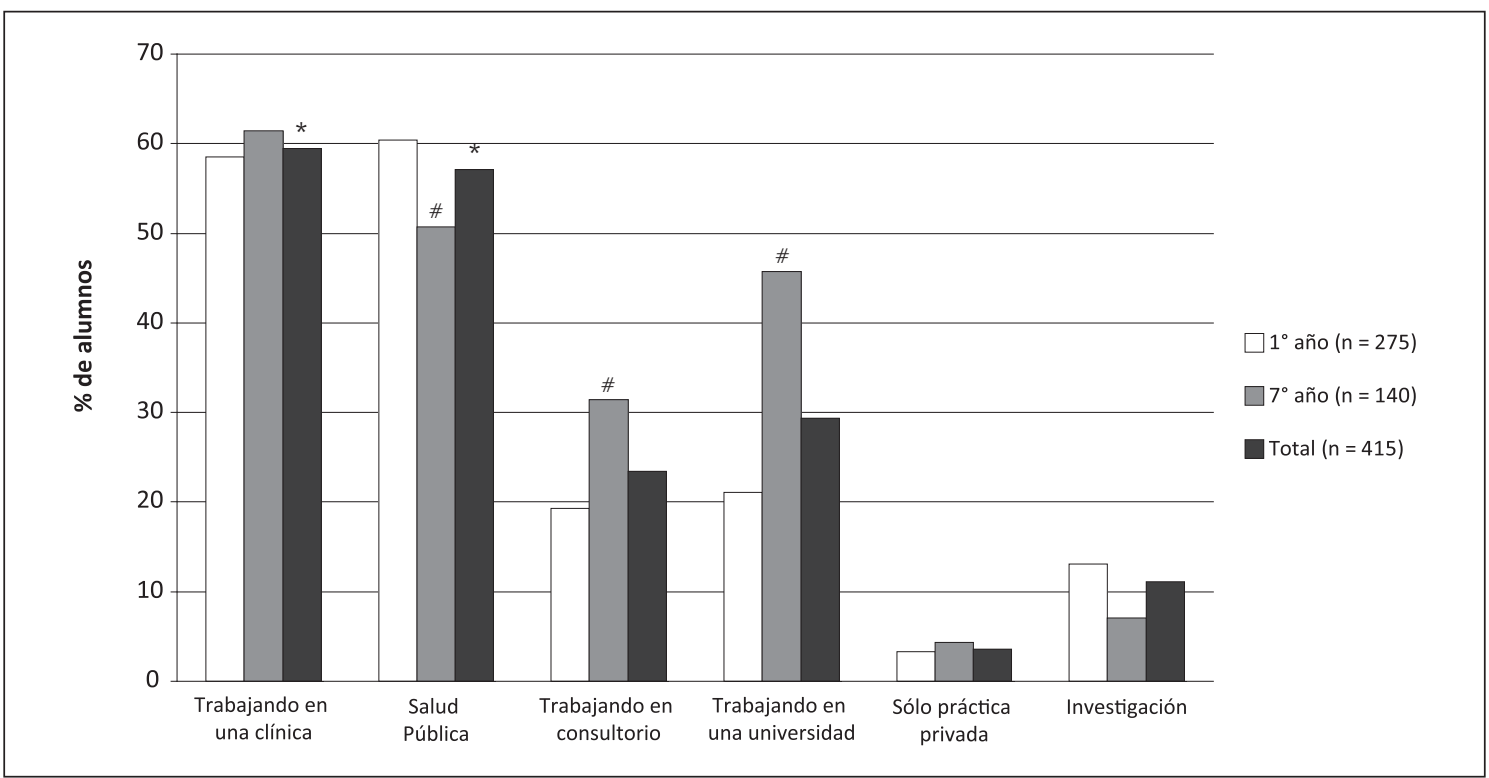

Figura 3. Proyección profesional de los alumnos de $1^{\circ}$ y $7^{\circ}$ año. ${ }^{*} p<0,05$ respecto a todas las otras preferencias por universidad y por curso; no significativas entre ellas. $\# p<0,05$ entre alumnos de $1^{\circ}$ y $7^{\circ}$ por ítem correspondiente.

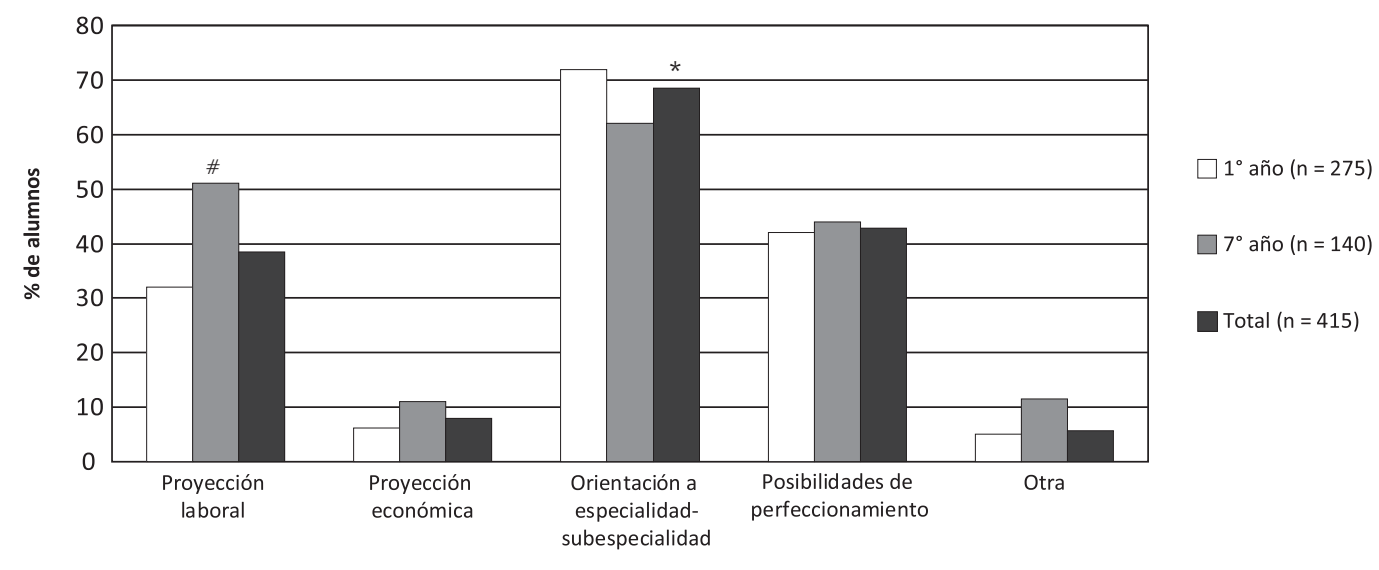

Figura 4. Información adicional que desearían recibir los estudiantes durante la carrera de medicina. * $p<0,05$ respecto a todas las otras preferencias. $\# p<0,05$ entre alumnos de $1^{\circ}$ y $7^{\circ}$ año respecto a Proyección Laboral. 
mento de interés sobre proyección económica en los alumnos de $7^{\circ}$ respecto a los de $1^{\circ}(16,1 \%$ y $3,9 \%),(\mathrm{p}<0,05)$.

En relación a la encuesta aplicada no se analizaron los datos extraídos de los antecedentes de los estudiantes y los relacionados con la elección de una especialidad o subespecialidad (Figura 1).

\section{Discusión}

Los resultados de este estudio muestran que el motivo principal del estudiante de medicina para ingresar a la carrera es el interés social seguidos por el interés por la ciencia y por lo académico. Si bien los órdenes de preferencia varían durante la carrera, son estos tres los que lideran las preferencias dejando atrás otros argumentos como status en la sociedad, buen puntaje en pruebas de selección, proyección económica, presión familiar, etc.

El aumento por el interés académico observado entre $1^{\circ}$ y $7^{\circ}$ año, no concuerda con un estudio de inicio de década en Estados Unidos de Norteamérica ${ }^{1}$. Este fenómeno probablemente se debe al sumergirse en un ambiente universitario estimulante, que permite al estudiante conocer e involucrarse en docencia e investigación, generando un incentivo para elegirlo como forma de desarrollo profesional. La diferencia con lo observado en Estados Unidos podrían atribuirse en parte a las motivaciones de una sociedad diferente a la nuestra, donde la decisión de la especialidad a seguir está muy ligada a la renta ${ }^{7}$. En contraste, destaca el bajo porcentaje de alumnos de $1^{\circ}$ año interesados en investigación, que no aumenta durante la carrera lo que concuerda con datos internacionales ${ }^{1,2}$. Esto sugiere que, dentro de la carrera académica, el interés va guiado hacia la docencia. Esta situación, se ha comenzado a revertir en los Estados Unidos de Norteamérica a partir de políticas de incentivos para formación de investigadores, que se comenzaron a aplicar a principios de la década pasada ${ }^{1}$.

Es destacable que al iniciar la carrera más de la mitad de los alumnos se proyecta trabajando en una clínica o en salud pública terciaria. Sin embargo, en $7^{\circ}$ disminuye el interés por trabajar en salud pública terciaria, a expensas de un aumento por atención de salud pública primaria, que es únicamente significativo en U3. Esto puede deberse a que en $7^{\circ}$ año se conoce de mejor forma la red de salud. Las diferencias por establecimiento, pueden basarse en el énfasis que le pudiera dar cada escuela de medicina, a las actividades de consultorio y al enfoque social de su formación.

Otro aspecto sorprendente es la baja proporción de alumnos que desean ser Médicos Generales de Zona, principalmente en las universidades privadas (3,3\% U1, 3,6\% U2, versus U3 20\%). Incentivar esta ocupación y otras que se refieran a atención primaria, podría ayudar a descentralizar los recursos de salud. Existe un gran interés en especialización y con la intención de mejorar su distribución existen programas en áreas deficientes con compromiso de retorno a algún servicio de salud de Chile ${ }^{8}$. Otra medida reciente ha sido la creación de los programas de "Título de Especialistas para la Atención Primaria (PTE-APS)", en que los médicos alternan la formación de especialista con estadías en consultorio ${ }^{8}$.

Creemos muy destacable la falta de motivación de más de un tercio de los alumnos de $7^{\circ}$ año. Dentro de esta misma pregunta existe un contraste entre las universidades privadas (U1, U2) y la universidad tradicional pública (U3). Es interesante analizar este fenómeno si pensamos que la mayoría de estos estudiantes ingresaron por motivación social y académica. Algo debe ocurrir durante la carrera para fomentar este desencanto. Los argumentos aludidos sumados al ambiente actual de gran competitividad más la necesidad de información que plantean los alumnos de medicina, son un factor a considerar. Áreas como futura especialización, posibilidades de perfeccionamiento y proyección laboral debieran ser abordadas de alguna forma durante el transcurso de la carrera. Sería interesante realizar otro estudio analizando si esta desmotivación persiste en médicos recibidos, y si es diferente de acuerdo a la elección laboral.

Las limitaciones del trabajo son inherentes a una encuesta de tipo transversal. En primer lugar las encuestas fueron respondidas por todos los alumnos a quienes se les entregó que representan $84 \%$ de los alumnos de $1^{\circ}$ año y $42,3 \%$ de los alumnos cursando $7^{\circ}$ año en las facultades descritas durante un año académico. La disminución en el porcentaje de alumnos de $7^{\circ}$ año, fue debida a la dificultad de entregar por mano la encuesta a cada estudiante, ya que en ese año se encuentran en rotaciones diversas. De antemano no elegimos el método del correo electrónico para enviar las encuestas pues nos pareció más improbable que se 
contestaran. En cuanto a la forma de las preguntas se observa un sesgo. Respecto a la motivación para estudiar medicina, en la encuesta no se especifica cuando se pregunta por salud pública, si corresponde a la práctica en un hospital o como médico salubrista, lo que podría haber confundido a los alumnos descartando esta alternativa. Respecto a las preguntas sobre especialización y subespecialización decidimos no incorporarlas al análisis. En el caso de elección de especialidad decidimos sólo analizar la respuesta de estudiantes de $7^{\circ}$ sobre "General de Zona", a pesar de no corresponder a una especialidad, ya que es una forma de acceder a la formación de especialista. Descartamos el análisis de la elección del resto de las especialidades/subespecialiades, en ambos cursos, por error en la oferta de alternativas. El instrumento tiene limitaciones, mayoritariamente respecto a su validación, pero a pesar de esto permite extraer información inicial importante sobre los intereses y motivaciones de los estudiantes de medicina en sus distintas etapas de formación.

En términos de educación en salud nos encontramos en un momento importante para definir el futuro de la medicina y la educación en salud de los próximos años. Existe un gran número de médicos en formación y una gran oferta universitaria. De acuerdo a sugerencias internacionales se estima que en un país debe existir al menos una escuela de medicina por cada millón de habitantes para cumplir con los requerimientos asistenciales de la población ${ }^{9,10}$. Chile cuenta con una relación médico/habitante (1:630), cifra que se enmarca dentro de los estándares de países desarrollados (1:213-714). Por lo que el problema de la falta de médicos del país, se refiere a la distribución de éstos ${ }^{3}$, centralizando los recursos en el área privada.

Los datos presentados contribuyen al conocimiento de un perfil del estudiante de medicina que ingresa y egresa de la carrera, de acuerdo a sus intereses y perspectivas. El conocer más detalladamente estos aspectos nos puede llevar a tomar medidas, como se ha hecho en otros países, que incentiven áreas deficitarias como la carrera académica y específicamente la investigación, tanto en rentabilidad personal como en recursos disponibles. De esta forma podremos prevenir la disminución excesiva de académicos e investigadores y así asegurar de buena forma la educación de la creciente masa de estudiantes que ingresan a estudiar medicina. Permitiendo una educación en salud de calidad, que mantenga a nuestro país con una medicina e investigación de excelencia.

Por último, creemos que es urgente crear un sistema de análisis de los intereses de los estudiantes de medicina de Chile, a nivel global, con encuestas estandarizadas y normadas, realizadas al comienzo y fin de la carrera (o examinar a los profesionales ya egresados), utilizando un sistema que se asemeje al que ya se utiliza en Estados Unidos de Norteamérica hace varios años. Es importante ya que esta información es fundamental para una buena intervención.

\section{Referencias}

1. Ley TJ, Rosenberg LE. The physician-scientist career pipeline in 2005: build it, and they will come. JAMA 2005; 294 (11): 1343-51.

2. Reck SJ, Stratman EJ, Vogel C, Mukesh BN. Assessment of residents loss of interest in academic careers and identification of correctable factors. Arch Dermatol 2006; 142 (7): 855-8.

3. Salazar C, Cardemil F, Peña J. Estado actual e implicancias de la acreditación de Escuelas de Medicina en Chile. Rev Med Chile 2009; 137 (8): 1126-7.

4. Sitio Web Oficial del Examen Único Nacional de Conocimientos en Medicina (EUNACOM). http://www. eunacom.cl/ [Consultado el martes 1 de marzo de 2011].

5. Graduation Questionnaire (GQ)-Previous Questionnaires. Association of American Medical Colleges (AAMC). https://www.aamc.org/data/gq/questionnaires/. [Consultado el miércoles 7 de octubre de 2009].

6. Matriculating Student Questionnaire (MSQ)-Previous Questionnaires. Association of American Medical Colleges (AAMC). https://www.aamc.org/data/msq/. [Consultado el miércoles 7 de octubre de 2009].

7. Julian K, Riegels NS, Baron RB. Perspective: creating the next generation of general internists: a call for medical education reform. Acad Med 2011; 86 (11): 1443-7.

8. Bases Concurso Especialidades Primarias, Egresados 2010. Diciembre 06, 2010, act. 03/01/2011. Escuela de Postgrado, Facultad de Medicina, Universidad de Chile. http://www.postgradomedicina.uchile.cl/med.portal? $\mathrm{nfpb}=$ true\&url=9857\&_pageLabel $=$ conUrl. [Consultado el martes 11 de enero de 2011].

9. Giaconi J. La educación médica privada en Chile. Rev Chil Pediatr 2002; 73: 339-40.

10. Goic A. Proliferación de escuelas de medicina en Latinoamérica: causas y consecuencias. Rev Med Chile 2002; 130 (8): 917-24. 